Publ. RIMS, Kyoto Univ.

14 (1978), 643-654

\title{
Immersion Analytique d'une Famille de Surfaces de Riemann Ouvertes
}

Par

\author{
Yasuichiro NISHIMURA*
}

\section{§. Introduction}

Soit $B=\{|z|<\lambda\}$ un polycylindre dans $C^{m}$ de $m$ variables complexes $z_{1}, \ldots, z_{m}(m \geqq 1)$, où $|z|=\operatorname{Max}\left\{{ }_{1} z_{i} \mid ; 1 \leqq i \leqq m\right\}$ et $\lambda>0$. Nous considérons une famille holomorphe de surfaces de Riemann ouvertes sur $B$. (Voir [7].) Elle est formée d'une variété analytique complexe $D$ de dimension $m+1$, et d'une application holomorphe surjective $\pi: D \rightarrow B$, telles que, pour tout $z \in B, D_{z}=\pi^{-1}(z)$ soit de dimension pure 1 et non compact. On pose ici les hypothèses suivantes:

$1^{\circ} D$ est une variété de Stein connexe.

$2^{\circ}$ La matrice fonctionnelle de $\pi$ est de rang $m$ partout dans $D$. Pour tout $z \in B, D_{z}$ est un ensemble analytique irréductible dans $D$.

$3^{\circ}$ Pour tout $z \in B, D_{z}$ est une surface de Riemann ouverte de type fini $(g, n)$, où $g$ est le genre de $D_{z}$, et $n$ est le nombre des composantes de frontière de $D_{z}$, et où $g$ et $n$ sont indépendants du choix de $z$.

Pour un nombre positif $r<\lambda$, on pose $B(r)=\{|z|<r\}, \overline{B(r)}=$ $\{|z| \leqq r\}, D(r)=\pi^{-1}(B(r))$ et $\overline{D(r)}=\pi^{-1}(\overline{B(r)})$. Cela posé, on peut énoncer le

Théorème 1. Supposons que la famille $(D, \pi, B)$ satisfait aux conditions $1^{\circ} \sim 3^{\circ}$. Alors, il existe un nombre positif $r<\lambda$ et une fonction holomorphe $h$ dans $D(r)$ tels que $\pi \times h: D(r) \rightarrow B(r) \times \mathbb{C}^{1}$ soit une immersion analytique. (C'est-à-dire que sa matrice fonctionnelle

* Communiqué par S. Nakano, juin le 16, 1977. Revu octobre le 24, 1977.

Section des Mathématiques, Faculté des Sciences, Université de Kyoto, Kyoto 606, Japon. 
soit de rang $m+1$ partout dans $D(r)$.

Ce théorème est une généralisation d'un résultat de Nishino [4] pp. 231-236, où il considérait le cas où $g=0$ et $n=1$.

Le but de cette note est de le démontrer selon la méthode de Gunning-Narasimhan [1]. Pour appliquer cette méthode à notre cas, nous préparons un théorème d'approximation —un théorème de type de Mergelyan-Bishop. (Voir §3.)

\section{§ 2. Construction des Domaines Qui Sont Equivalents aux Domaines Produits}

Nous utilisons le mot $X$-convexe comme ci-dessous. Soit $X$ une variété de Stein. Un domaine $G$ dans $X$ est dit $X$-convexe, si, pour tout compact $K$ dans $G, \hat{K} \Subset G$ où $\hat{K}$ signifie l'envellope d'holomorphie de $\hat{K}$ par rapport à $X$. Un compact $K$ dans $X$ est dit $X$-convexe, si $\hat{K}=K$.

Nous allons construire deux domaines qui joueront un rôle essentiel dans nos études.

Lemme 1. Soit donnée une famille $(D, \pi, B)$ satisfaisant aux conditions $1^{\circ} \sim 3^{\circ}$. Alors, il existe un nombre positif $\rho(<\lambda)$, un domaine $D^{\prime}$ dans $D(\rho)$ et une application holomorphe $v: D^{\prime} \rightarrow D_{o}^{\prime}$ (ou $D_{z}^{\prime}=D^{\prime} \cap D_{x}$ et $o=(0, \ldots, 0) \in B)$ qui satisfont aux conditions suivantes:

$4^{\circ}$ (i) $\pi \times v: D^{\prime} \rightarrow B(\rho) \times D_{o}^{\prime}$ est biholomorphe;

(ii) Pour tout $z \in B(\rho), D_{z}^{\prime}$ est connexe, de type $(g, n)$, délimité par $n$ courbes de Jordan simples fermées;

(iii) $D_{z}^{\prime} \Subset D_{z}$ et $D_{z}^{\prime}$ est $D_{z}$-convexe.

En effet, il existe une immersion $f: D_{o} \rightarrow C^{1}$ d'après [1]. Comme $D$ est une variété de Stein, il existe une fonction holomorphe $\hat{f}$ dans $D$ telle que $\left.\hat{f}\right|_{D_{o}}=f$. Comme $d f \neq 0$ sur $D_{o}$, à chaque point $p \in D_{o}$, on peut associer un voisinage $V(p)$ de $p$ tel que $\pi \times \hat{f}: V(p) \rightarrow B\left(\rho_{p}\right) \times C_{p}$ soit biholomorphe, où $\rho_{p}>0$ et $C_{p}$ est un disque dans le plan. Alors, $f^{-1} \circ \hat{f}$ définit une rétraction analytique $v_{p}$ de $V(p)$ dans $D_{o}$ dont la 
restriction sur $V(p) \cap D_{z}$ est une application biholomorphe de $V(p) \cap D_{z}$ sur $V(p) \cap D_{o}$, quel que soit $z \in B\left(\rho_{p}\right)$. Soit $U$ la réunion de tous ces voisinages $V(p)$ pour $p \in D_{o}$ et soit $v: U \rightarrow D_{o}$ l'application holomorphe définie par $v_{p}$ dans $V(p)$. Nous allons montrer qu'elle est bien définie. Soit $q \in V(p)$, alors $F_{p}=V(p) \cap \hat{f}^{-1}(\hat{f}(q))$ se projette biunivoquement sur $B\left(\rho_{p}\right)$ par l'application $\left.\pi\right|_{F_{p}}$. On considère l'application holomorphe $g=\left(\left.\pi\right|_{F_{p}}\right)^{-1}: B\left(\rho_{p}\right) \rightarrow D$. Si $q \in V(p) \cap V\left(p^{\prime}\right)$, on a comme ci-dessus $g^{\prime}=\left(\left.\pi\right|_{F_{p^{\prime}}}\right)^{-1}: B\left(\rho_{p^{\prime}}\right) \rightarrow D$ pour $V\left(p^{\prime}\right)$. Puisque les applications holomorphes $g$ et $g^{\prime}$ coincident dans un voisinage de $\pi(q), g \equiv g^{\prime}$ dans $B\left(\rho_{p}\right) \cap B\left(\rho_{p^{\prime}}\right)$. On a donc $g(o)=g^{\prime}(o) \in D_{o}$ et ce point coincide avec $v(q)$. Ceci indique que $v$ est bien définie. La restriction de $v$ sur toute fibre $U \cap D_{z}$ non vide est injective. En effet, si ce n'est pas vrai, il y a deux points $q, q^{\prime}$ dans $D_{z}$, appartenant à $V(p), V\left(p^{\prime}\right)$ respectivement, tels que $v(q)=v\left(q^{\prime}\right)$. On considère comme cidessus les applications holomorphes $g: B\left(\rho_{p}\right) \rightarrow D$ pour $V(p)$ et $g^{\prime}: B\left(\rho_{p^{\prime}}\right) \rightarrow D$ pour $V\left(p^{\prime}\right)\left(g^{\prime}=\left(\left.\pi\right|_{F_{p^{\prime}}}\right)^{-1}\right.$ où $\left.F_{p^{\prime}}=V\left(p^{\prime}\right) \cap \hat{f}^{-1}\left(f\left(q^{\prime}\right)\right)\right)$. Ces applications holomorphes $g$ et $g^{\prime}$ conicident dans un voisinage de $o \in B \in C^{m}$ puisque $v(q)=v\left(q^{\prime}\right)$. Donc, $g \equiv g^{\prime}$ dans $B\left(\rho_{p}\right) \cap B\left(\rho_{p^{\prime}}\right)$. On a $g(z)=g^{\prime}(z)$. D'autre part $g(z)=q$ et $g^{\prime}(z)=q^{\prime}$, une contradiction. Maintenant, il existe un domaine $D_{o}^{\prime} \Subset D_{o}$, de type $(g, n)$, délimité par courbes de Jordan simples fermées, tel que $D_{o}^{\prime}$ soit $D_{o}$-convexe. En prenant un nombre positif $\rho$ suffisamment petit on obtient le domaine $D^{\prime}$ qui satisfait aux conditions $4^{\circ}$ avec l'application $v$ restreinte à $D^{\prime}$.

C. Q. F.D.

Lemme 2. Soit donnée une famille $(D, \pi, B)$ satisfaisant aux conditions $1^{\circ} \sim 3^{\circ}$ et soient donnés un nombre $\rho>0$, un domaine $D^{\prime}$ et une application $v$, qui satisfont aux conditions $4^{\circ}$. Alors, il existe un domaine $D^{\prime \prime}$ dans $D^{\prime}$ et une fonction plurisousharmonique $\Phi$ dans $D(\rho)$ ayant les propriétés suivantes (On pose $D^{\alpha}=\{p \in D(\rho) \mid \Phi(p)<\alpha\}$ et $D_{z}^{\prime \prime}=$ $\left.D^{\prime \prime} \cap D_{z}\right)$ :

$5^{\circ}$ (i) $\Phi$ est strictement plurisousharmonique et complète dans $D(\rho) .{ }^{(1)}$

(1) Soit $\Phi(p)$ une fonction strictment plurisousharmonique (de classe $C^{\infty}$ ) dans une variété analytique complexe $X$. Si, pout tout nombre réel $\alpha$, on $X^{\alpha}=\{p \in X \mid \Phi(p)<\alpha\} \Subset X$, on dit que $\Phi$ est complète. 
(ii) Pour tout $z \in B(\rho), D_{z}^{\prime \prime}$ est connexe, de type $(g, n)$, delimité par courbes de Jordan simples fermées, $D_{z}^{\prime \prime} \Subset D_{z}^{\prime}$, et $D_{z}^{\prime \prime}$ est $D_{z}^{\prime}$-convexe. $\pi \times v: D^{\prime \prime} \rightarrow B(\rho) \times D_{o}^{\prime \prime}$ est biholomorphe.

(iii) Il existe un nombre positif $\rho_{1}(<\rho)$ et un nombre réel $\alpha$ tels que, pour tout $z \in \overline{B\left(\rho_{1}\right)}$, on ait $D_{z}^{\prime \prime} \Subset D_{z} \cap D^{\alpha} \Subset D_{z}^{\prime}$.

En effet, on peut prendre un domaine $D_{o}^{\prime \prime} \Subset D_{o}^{\prime}$ qui est de type $(g, n)$, delimité par courbes de Jordan simples fermées, et $D_{o}^{\prime}$-convexe. Soit $D^{\prime \prime}=(\pi \times v)^{-1}\left(B(\rho) \times D_{o}^{\prime \prime}\right)$. Alors, $\overline{D_{o}^{\prime \prime}}$ ( $\_$indique la fermeture dans $D(\rho))$ est $D(\rho)$-convexe. En effet, pour un point $p \in D_{\text {o }}$ tel que $p \notin \overline{D_{o}^{\prime \prime}}$, il existe une fonction holomorphe $g$ sur $D_{o}$ telle que $|g(p)|>$ $\operatorname{Max}\left\{|g(q)| \mid q \in \overline{D_{o}^{\prime \prime}}\right\}$, puisque $\overline{D_{o}^{\prime \prime}}$ est $D_{o}$-convexe. Comme $D(\rho)$ est une variété de Stein, on peut trouver une fonction holomorphe $\hat{g}$ dans $D(\rho)$ telle que $\left.\hat{g}\right|_{D_{o}}=g$. On a alors $|\hat{g}(p)|>\operatorname{Max}\left\{|\hat{g}(q)| \mid q \in \overline{D_{o}^{\prime \prime}}\right\}$. Pour un point $p \in D(\rho)$ tel que $\pi(p) \neq 0$, au moins une des coordonnées $z_{i}$ satisfait à $\left|z_{i}(p)\right|>0$, ce qui montre que $\overline{D_{o}^{\prime \prime}}$ est $D(\rho)$-convexe. Comme $D(\rho)$ est une variété de Stein, il existe une fonction plurisousharmonique $\Phi$ ayant la propriété $5^{\circ}$ (i) et admettant un nombre réel $\alpha$ tel que $D_{o}^{\prime \prime} \Subset D^{\alpha} \Subset D^{\prime}$. (Voir [2] p. 106.) Si l'on prend un nombre $\rho_{1}>0$ assez petit, la condition $5^{\circ}$ (iii) sera remplie.

C. Q. F. D.

Dans les sections suivantes, nous fixons les nombres positifs $\rho$ et $\rho_{1}$, les domaines $D^{\prime}$ et $D^{\prime \prime}$, la fonction plurisousharmonique $\Phi$, et le nombre réel $\alpha$ qui satisfont aux conditions $4^{\circ}$ et $5^{\circ}$.

\section{§3. Théorème d'Approximation}

Pour nous appuyer sur la méthode de Gunning-Narasimhan [1], nous avons besoin de généraliser le théorème de Mergelyan-Bishop.

Lemme 3. Soit $R$ une surface de Riemann ouverte, et soit $K$ un ensemble compact dans $R$ tel que $K$ soit $R$-convexe. Soit $u(z, p)$ une fonction continue dans $B\left(\rho_{2}\right) \times K$ ayant les propriétés suivantes:

(i) Si l'on fixe z, elle est holomorphe par rapport à $p$ dans l'inté- 
rieur de $K$.

(ii) Si l'on fixe $p$, elle est holomorphe par rapport à $z$ dans $B\left(\rho_{2}\right)$. Alors, on peut faire approcher de $u(z, p)$ uniformément sur $\overline{B(r)} \times K$ par des fonctions holomorphes dans $B\left(\rho_{2}\right) \times R$, quel que soit le nombre positif $r$ plus petit que $\rho_{2}$.

En effet, la série de Taylor $u(z, p)=\sum_{n_{1}, \cdots, n_{m}=0}^{\infty} a_{n_{1} \cdots n_{m}}(p) z_{1}^{n_{1}} \cdots z_{m}^{n_{m}}$ converge uniformément sur $\overline{B(r)} \times K . \quad a_{n_{1} \cdots n_{m}}(p)$ sont continues sur $K$ et holomorphes dans l'intérieur de $K$. Alors, pour un nombre positif quelcoque $\varepsilon$, il existe un ensemble fini $J$ d'indices $n=\left(n_{1}, \ldots, n_{m}\right)$ tel que $\left|u_{J}-u\right|<\frac{\varepsilon}{2}$ sur $\overline{B(r)} \times K$, où $u_{J}(z, p)=\sum_{n \in J} a_{n}(p) z_{1}^{n_{1} \cdots} z_{m}^{n_{m}} \cdot \mathrm{A}$ chaque $n \in J$, on fait correspondre $\varepsilon_{n}>0$ de façon que $\sum_{n \in J} \varepsilon_{n} r^{n_{1}+\cdots+n_{m}}<\frac{\varepsilon}{2}$. D'après le théorème de Mergelyan-Bishop, on peut trouver une fonction $f_{n}(p)$ holomorphe dans $R$ telle que $\left|f_{n}(p)-a_{n}(p)\right|<\varepsilon_{n}$ sur $K(n \in J)$. La fonction $f(p)=\sum_{n \in J} f_{n}(p) z_{1}^{n_{1}} \cdots z_{m}^{n_{m}}$ est holomorphe dans $B\left(\rho_{2}\right) \times R$ et satisfait à $\left|u_{J}-f\right|<\frac{\varepsilon}{2}$ sur $\overline{B(r)} \times K$, donc on a $|f-u|<\varepsilon \operatorname{sur} \overline{B(r)} \times K$. C. Q. F. D.

Théorème 2. Soit $K_{o}$ un ensemble compact dans $D_{o}^{\prime \prime}$ tel que $K_{\text {o }}$ soit $D_{o}^{\prime}$-convexe. Soit $u(z, p)$ une fonction continue dans $B\left(\rho_{2}\right) \times K_{o}$ $\left(0<\rho_{2}<\rho\right)$ ayant les propriétés suivantes :

(i) Si l'on fixe $p$, elle est holomorphe par rapport à $z$ dans $B\left(\rho_{2}\right)$.

(ii) Si l'on fixe $z$, elle est holomorphe par rapport à $p$ dans l'intérieur de $K_{0}$.

Alors, on peut faire approcher de la fonction $u_{\circ}(\pi \times v)$ uniformément sur le compact $K=\bigcup_{z \in \overline{B(r)}} v^{-1}\left(K_{o}\right) \cap D_{z}$ par des fonctions holomorphes dans $D(\rho)$, quel que soit le nombre positif $r<\operatorname{Min}\left(\rho_{1}, \rho_{2}\right)$.

En effet, pour un nombre positif quelconque $\varepsilon$, il existe, d'après le lemme 3, une fonction holomorphe $f$ dans $B\left(\rho_{2}\right) \times D_{o}^{\prime}$ telle que $|f-u|<\frac{\varepsilon}{2}$ sur $\overline{B(r)} \times K_{o}$, quel que soit le nombre positif $r<\rho_{2}$. D'autre part, pour tout nombre positif $\rho^{\prime} \leqq \rho, D\left(\rho^{\prime}\right) \cap D^{\alpha}$ est $D(\rho)$ - 
convexe. (Voir les conditions $5^{\circ}$.) D'après le théorème de Weil-Oka, pour toute fonction holomorphe $h$ sur $D\left(\rho^{\prime}\right) \cap D^{\alpha}$, on peut faire approcher de $h$ uniformément sur tout compact dans $D\left(\rho^{\prime}\right) \cap D^{\alpha}$ par des fonctions holomorphes dans $D(\rho)$. Si, pour tout nombre $r$ tel que $0<r<\operatorname{Min}\left(\rho_{1}, \rho_{2}\right)$, on prend le nombre $\rho^{\prime}$ de manière que $r<\rho^{\prime}<\operatorname{Min}\left(\rho_{1}, \rho_{2}\right)$, on obtient $K \Subset D\left(\rho^{\prime}\right) \cap D^{\alpha} \Subset D^{\prime}\left(\rho_{2}\right)$. (Voir les conditions $5^{\circ}$.) Alors, pour la fonction holomorphe $f \circ(\pi \times v) \mid D\left(\rho^{\prime}\right) \cap D^{\alpha}$, on peut trouver une fonction holomorphe $g$ dans $D(\rho)$ telle que $|f \circ(\pi \times v)-g|<\frac{\varepsilon}{2}$ sur $K$. Alors $|g-u \circ(\pi \times v)|<\varepsilon$ sur $K$.

C. Q. F. D.

\section{§4. Démonstration du Théorème 1}

D'abord, montrons le

Lemme 4. $\mathrm{H}^{2}(D(\rho), Z)=0$.

Il suffit de montrer $\mathrm{H}_{2}(D(\rho), \boldsymbol{Z})=0$ et $\mathrm{H}_{1}(D(\rho), \boldsymbol{Z})=\mathbb{Z}^{2 \boldsymbol{E}+n-1}$, d'après le théorème des coefficients universels. Pour cela, on va montrer que, pour tout ensemble compact $K$ dans $D(\rho)$, il existe un ensemble compact $E$ et une retraction continue $\tilde{v}: E \rightarrow E_{o}$ (ou $E_{o}=E \cap D_{o}$ ) tels que : (i) $K \subset E \subset D(\rho)$; (ii) $\pi(E)=\overline{B\left(\rho^{\prime}\right)}$ (ou $0<\rho^{\prime}<\rho$ ); (iii) $\pi \times \tilde{v}$ : $E \rightarrow \overline{B\left(\rho^{\prime}\right)} \times E_{\text {o }}$ soit un homéomorphisme. En effet, on prend $\rho^{\prime}$ de façon que $\pi(K) \subset B\left(\rho^{\prime}\right)$ et $0<\rho^{\prime}<\rho$. Soit $\beta$ un nombre réel suffisamment grand pour que $D_{z}^{\prime \prime} \Subset D_{z}^{\beta}$ pour tout $z \in \overline{B\left(\rho^{\prime}\right)}$ et que $K \subset \bigcup_{z \in B\left(\rho^{\prime}\right)} D_{z}^{\beta}$, où $D_{z}^{\beta}$ est la composante connexe de $D^{\beta} \cap D_{z}$, qui contient $D_{z}^{\prime \prime}$. ( $D^{\beta}$ est défini par $\Phi<\beta$ pour la fonction plurisousharmonique $\Phi$ dans $D(\rho)$ donnée dans la condition $5^{\circ}$.) Comme $D_{z}^{\prime \prime}$ est $D_{z}$-convexe, que $D_{z}^{\prime \prime}$ et $D_{z}$ sont de type $(g, n)$, et que $D_{z}^{\beta}$ est $D_{z}$-convexe (Voir Nishino [6] p. 245), $D_{z}^{\beta}$ est de type $(g, n)$. $D_{z}^{\beta} \backslash D_{z}^{\prime \prime}$ se décompose en $n$ composantes connexes $R_{z}^{1}, \ldots, R_{z}^{n}$, chacune desqueiles est analytiquement équivalente à un domaine annulaire. Soit $\gamma_{z}^{j}$ la composante connexe du bord de $R_{z}^{j}$, qui fait partie du bord $D_{z}^{\prime \prime}$, et soit $\delta_{z}^{j}$ l'autre composante connexe du bord de $R_{z}^{j}$, qui fait alors partie du bord de $D_{z}^{\beta}(j=1, \ldots, n)$. On construit une fonction $h_{z}^{j}$, qui est continue sur $\overline{R_{z}^{j}}$, harmonique 
dans $R_{z}^{j}$, et qui prend la valeur 0 sur $\gamma_{z}^{j}$ et la valeur 1 sur $\delta_{z^{*}}^{j}$ Si on définit une fonction $h^{j}$ dans $\underset{z \in \bar{B}\left(\rho^{\prime}\right)}{\bigcup} \overline{R_{z}^{j}}$ par $\left.h^{j}\right|_{R_{z}^{j}}=h_{z}^{j}$, $h^{j}$ est continue par rapport à plusieurs variables. (Voir [6] p. 265.) On prend un nombre $a(0<a<1)$ de façon que $K \Subset \cup\left(\overline{D_{z}^{\prime \prime}} \cup T_{z}^{1} \cup \ldots \cup T_{z}^{n}\right)$, où $T_{z}^{j}=\left\{p \in R_{z}^{j}\right.$

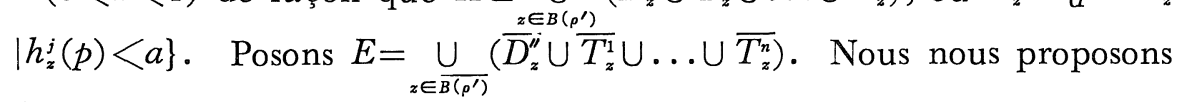
de montrer que $E$ satisfait à la condition (iii) ci-dessus. On prend pour chaque $j=1, \ldots, n$ un point $Q_{o}^{j} \in D_{o}^{\prime} \backslash D_{o}^{\prime \prime}$ tel que $Q_{z}^{j}=v^{-1}\left(Q_{o}\right) \cap D_{z} \in T_{z}^{j}$. Notons $* h_{z}^{j}$ la fonction harmonique conjuguée de $h_{z}^{j}$ dans $R_{z}^{j}$, dont l'une des branches prend la valeur 0 au point $Q_{z}^{j}$. $C^{j}(z)$ étant la variation de $* h_{z}^{j}$ le long du cycle $\delta_{z}^{j}$ orienté de la façon habituelle, la fonction $g_{z}^{j}=\exp \left(2 \pi\left(h_{z}^{j}+\sqrt{-1} * h_{z}^{j}\right) / C^{j}(z)\right)$ est uniforme et holomorphe dans $R_{z}^{j}$, où l'on remarque que $C^{j}(z)$ est positive et continue par rapport à $z$. Alors $g_{z}^{j}$ transforme homéomorphiquement $\overline{T_{z}^{j}}$ sur le domaine annulaire dans le plan, de centre 0 et de rayons 1 et $r^{j}(z)=\exp \left(2 \pi a / C^{j}(z)\right)$. A l'aide de ces fonctions $g_{z}^{j}$, on peut facilement prolonger la retraction $v$ restrainte à $\overline{D^{\prime \prime}\left(\rho^{\prime}\right)}$ en une retraction continue $\tilde{v}: E \rightarrow E_{o}$ telle que $\pi \times \tilde{v}: E \rightarrow \overline{B\left(\rho^{\prime}\right)} \times E_{o}$ soit un homéomorphisme. L'existence d'une telle retraction montre bien qu'on a l'isomorphisme $\mathrm{H}_{i}(D(\rho), \boldsymbol{Z}) \approx \mathrm{H}_{i}\left(D_{o}, \boldsymbol{Z}\right)$ pour tout $i$. D'où, $\mathrm{H}_{2}(D(\rho), \boldsymbol{Z})=0$ et $\mathrm{H}_{1}(D(\rho), \boldsymbol{Z})=\mathbb{Z}^{2 \boldsymbol{g}+n-1}$.

C. Q. F. D.

Maintenant, prenons un point $O_{o} \in D_{o}^{\prime \prime}$ et une coordonnée locale $w_{o}$ en $O_{o}$, définie dans un voisinage $\mathrm{U}_{o}$ de $O_{o}$ dans $D_{o}^{\prime \prime}$. Posons $U=v^{-1}\left(U_{o}\right) \subset D(\rho), w=w_{o} \circ\left(\left.v\right|_{U}\right), U_{z}=U \cap D_{z}$ et $O_{z}=v^{-1}\left(O_{o}\right) \cap D_{z}$ pour $z \in B(\rho)$ et fixons-les une fois pour toutes. Alors, $z_{1}, \ldots, z_{m}$, w forment un système de coordennées dans $U$. Soit donnée pour chaque $z \in B(\rho)$ une différentielle holomorphe $\omega_{z}$ sur $D_{z}$ tout entier, qui ne s'annule nulle part. On peut écrire $\omega_{z}=a(z, w) d w$ dans $U_{z}$. Lorsque $a(z, w)$ est holomorphe dans $U$ par rapport aux $z_{1}, \ldots, z_{m}$ et $w$, on appelle $\omega=\left(\omega_{z}\right)_{z \in B(\rho)}$ famille holomorphe de différentielles non nulles dans $D(\rho)$.

Nous nous proposons de montrer le

Lemme 5. Il existe une famille holomorphe de différentielles non 
nulles dans $D(\rho)$.

En effet, pour tout point $p$ de $D(\rho)$, il existe un voisinage $V$ de $p$ et une fonction holomorphe $u$ dans $V$ telles que $z_{1}, \ldots, z_{m}, u$ forment un système de coordonnées locales dans $V$ et qu'elles transforment $V$ en un polycylindre $\left\{z \in C^{m}|| z-\pi(p) \mid<\varepsilon\right\} \times\left\{w \in C|| w \mid<\varepsilon^{\prime}\right\}$ de la façon biholomorphe. $\left.d u\right|_{D_{z}}$ est alors une différentielle holomorphe sur $D_{z} \cap V$, qui ne prend pas de zéro. Recouvrons $D(\rho)$ d'un nombre dénomblable de tels vcisinages $V_{j}$ avec $u_{j}$. On suppose en particulier $\left(V_{1}, u_{1}\right)=(U, w)$ pris au début. Si $V_{i} \cap V_{j} \neq \phi$, on a $z_{1}=z_{1}, \ldots, z_{m}=z_{m}$, $u_{i}=\phi_{i j}\left(z, u_{j}\right)$ dans $V_{i} \cap V_{j}$. Le déterminant fonctionnel de cette transformation est

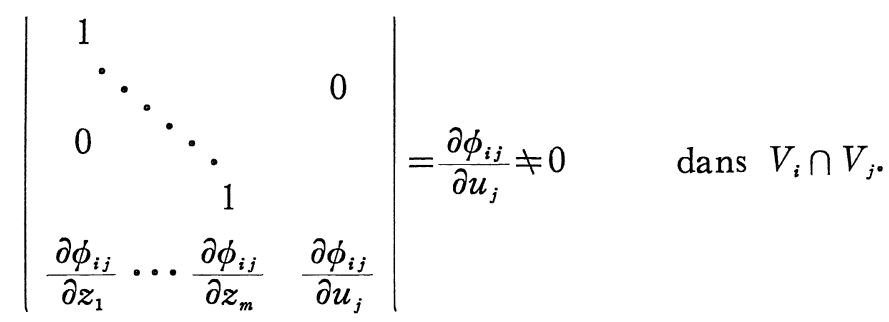

Les $\left(V_{i} \cap V_{j}, \frac{\partial \phi_{i j}}{\partial u_{j}}\right)$ définissent un élément de $\mathrm{H}^{1}\left(D(\rho), O^{*}\right)$. Comme $D(\rho)$ est une variété de Stein et que $\mathrm{H}^{2}(D(\rho), \mathbb{Z})=0$ d'après le lemme 4, on a $\mathrm{H}^{1}\left(D(\rho), O^{*}\right)=0$. ([2] p. 181.) Alors il existe un système de $a_{j} \in \mathrm{H}^{0}\left(V_{j}, O^{*}\right)$ tel que $a_{j} / a_{i}=\frac{\partial \phi_{i j}}{\partial u_{j}}$ dans $V_{i} \cap V_{j}$. Si l'on pose $\omega_{z}=\left.a_{j} d u_{j}\right|_{D_{z}}$ sur $V_{j} \cap D_{z}, \omega_{z}$ est une différentielle holomorphe sur $D_{z}$, qui ne s'annulle nulle part. Dans $U$, on a $\omega_{z}=a_{1}(z, w) d w$ où $a_{1}(z, w)$ est holomorphe par rapport à $z$ et $w$. $\quad$ C. Q. F. D.

Lemme 6. Soit $f(z, t)$ une fonction continue dans $B(\rho) \times[a, b]$ $\grave{a}$ valeurs complexes, et, si l'on fixe $t$, holomorphe par rapport à $z$. Soit c un nombre complexe quelconque. Alors, il existe un nombre réel positif $\rho_{2}<\rho$ et une fonction $g(z, t)$ ayant les propriétés suivantes:

(i) $g(z, t)$ est continue dans $\overline{B\left(\rho_{2}\right)} \times(a, b)$. Si l'on fixe $t$, elle est holomorphe sur $\overline{B\left(\rho_{2}\right)}$.

(ii) Son support est compact dans $\overline{B\left(\rho_{2}\right)} \times(a, b)$. 
(iii) Pour tout $z \in \overline{B\left(\rho_{2}\right)}$, on $a \int_{a}^{b} e^{f(z, t)+g(z, t)} d t=c$ et $\int_{a}^{b} g(z, t) e^{f(z, t)+g(z, t)} d t$ $\neq 0$.

Le démonstration sera partagée en trois parties.

1. Soient $a_{0}$ et $b_{0}$ deux nombres réels tels que $a<a_{0}<b_{0}<b$. Nous allons construire pour un certain nombre réel positif $\rho^{\prime}<\rho$ une fonction $u(z, t)$ ayant les propriétés suivantes: (i) $u(z, t)$ est une fonction en escalier par rapport à $t$, si l'on fixe $z$. Elle est holomorphe sur $\overline{B\left(\rho^{\prime}\right)}$, si l'on fixe $t$. (ii) Son support est contenu dans $\overline{B\left(\rho^{\prime}\right)} \times\left[a_{0}, b_{0}\right]$. (iii) Pour tout $z \in \overline{B\left(\rho^{\prime}\right)}$, on a $\int_{a}^{b} e^{f(z, t)+u(z, t)} d t=c$ et $\int_{a}^{b} u(z, t) e^{f(z, t)+u(z, t)} d t$ $\neq 0$. En effet, on prend des nombres $c_{0}$, $d_{0}$ tel que $a_{0}<c_{0}<d_{0}<b_{0}$. On pose, pour une variable complexe $d$ et pour deux variables réeles $\sigma$ et $\tau$,

$$
X\left(z, e^{d}, \sigma, \tau\right)=c-\mu\left(z ; a, a_{0}\right)-\mu(z ; \sigma, \tau)-\mu\left(z ; b_{0}, b\right)-e^{d} \mu(z ;
$$
$\left.a_{0}, \sigma\right)$, où $\mu(z ; \alpha, \beta)=\int_{\alpha}^{\beta} e^{f(z, t)} d t$. On peut prendre, pour $z=o=$ $(0, \ldots, 0)$, deux nombres réels $a_{1} \in\left(a_{0}, c_{0}\right)$ et $b_{1} \in\left(d_{0}, b_{0}\right)$ tels qu'on ait $\mu\left(o ; a_{0}, a_{1}\right) \neq 0, \mu\left(o ; b_{1}, b_{0}\right) \neq 0$ et que $d e^{d} \mu\left(o ; a_{0}, a_{1}\right)+X\left(o, e^{d}, a_{1}, b_{1}\right)$ $\log \left\{X\left(o, e^{d}, a_{1}, b_{1}\right) / \mu\left(o ; b_{1}, b_{0}\right)\right\}$ ne soit pas constante par rapport à la variable $d$. On peut prendre aussi un nombre complexe $k$ tel que $X\left(o, e^{k}, a_{1}, b_{1}\right) \neq 0$ et $k e^{k} \mu\left(o ; a_{0}, a_{1}\right)+X\left(o, e^{k}, a_{1}, b_{1}\right) \log \left\{X\left(o, e^{k}, a_{1}, b_{1}\right) /\right.$ $\left.\mu\left(o ; b_{1}, b_{0}\right)\right\} \neq 0$, en prenant une certaine branche du logarithme en $d=k$. On fixe les nombres $a_{1}, b_{1}$, et $k$, et cette branche du logarithme. Alors, il existe un nombre positi $\rho^{\prime}<\rho$ tel que, pour tout $z \in \overline{B\left(\rho^{\prime}\right)}$, on ait $\mu\left(z ; a_{0}, a_{1}\right) \neq 0, \quad \mu\left(z ; b_{1}, b_{0}\right) \neq 0, \quad X\left(z, e^{k}, a_{1}, b_{1}\right) \neq 0$ et $k e^{k} \mu\left(z ; a_{0}, a_{1}\right)+X\left(z, e^{k}, a_{1}, b_{1}\right) \log \left\{X\left(z, e^{k}, a_{1}, b_{1}\right) / \mu\left(z ; b_{1}, b_{0}\right)\right\} \neq 0$. Maintenant, on pose

$$
u(z ; t)=\left\{\begin{array}{lr}
k & \text { dans } \overline{B\left(\rho^{\prime}\right)} \times\left[a_{0}, a_{1}\right] \\
\log \left\{X\left(z, e^{k}, a_{1}, b_{1}\right) / \mu\left(z ; b_{1}, b_{0}\right)\right\} & \text { dans } \overline{B\left(\rho^{\prime}\right)} \times\left[b_{1}, b_{0}\right] \\
0 & \text { dans } \overline{B\left(\rho^{\prime}\right)} \times\left\{\left(a, a_{0}\right) \cup\left(a_{1}, b_{1}\right) \cup\left(b_{0}, b\right)\right\}
\end{array}\right.
$$

Nous voyons aussitôt qu'elle a les propriétés (i), (ii) et nous pouvons vérifier (iii) par un calcul direct.

2. Pour chaque point de discontinuité $t=a_{0}, a_{1}, b_{1}, b_{0}$ en modifiant la fonction $u(z, t)$ d'une façon linéaire par rapport à $t$ dans 
un intervalle ouvert contenant le point de discontinuité et devenant infiniment petit pour $\nu \rightarrow \infty$, on obtient aussitôt une suite de fonctions $\left\{g_{\nu}(z, t)\right\}_{\nu=1}^{\infty}$ ayant les propriétés suivantes: (i) Elles sont continues dans $\overline{B\left(\rho^{\prime}\right)} \times(a, b)$; Elles sont holomorphes dans $\overline{B\left(\rho^{\prime}\right)}$, si l'on fixe $t$; (ii) Leurs supports sont contenus dans un compact dans $\overline{B\left(\rho^{\prime}\right)} \times$ $(a, b)$, indépendant de $\nu$; (iii) Elles sont bornées uniformément; (iv) $g_{v}(z, t)$ convergent vers $u(z, t)$ uniformément sur tout compact dans $\overline{B\left(\rho^{\prime}\right)} \times T$, où $T=(a, b) \backslash\left\{a_{0}, a_{1}, b_{1}, b_{0}\right\}$.

3. En prenant une variable complexe $s$, on pose $\phi(s, z)=$ $\int_{a}^{b} e^{f(z, t)+s u(z, t)} d t$ et $\phi_{\nu}(s, z)=\int_{a}^{b} e^{f(z, t)+s g_{\nu}(z, t)} d t$. Ces fonctions sont holomorphes dans $\mathbb{C}^{1} \times B\left(\rho^{\prime}\right) . \quad \phi_{\nu}(s, z)$ convergent vers $\phi(s, z)$ uniformément sur tout compact dans $\mathbb{C}^{1} \times B\left(\rho^{\prime}\right)$. En vertu de la propriété (iii) de $u(z, t), \phi(1, z)=c$ et $\frac{\partial \phi}{\partial s}(1, z) \neq 0$ dans $B\left(\rho^{\prime}\right)$. On prend un nombre positif $\rho_{2}<\rho^{\prime}$. D'après le théorème de Rouché, on peut trouver un nombre entier $\nu$ suffisamment grand et une fonction holomorphe $s(z)$ sur $\overline{B\left(\rho_{2}\right)}$ ayant des valeurs assez voisines de 1 , de manière que $\phi_{\nu}(s(z), z)=c$ et $\frac{\partial \phi_{\nu}}{\partial s}(s(z), z) \neq 0$ dans $\overline{B\left(\rho_{2}\right)}$. Alors, la fonction $g(z, t)=s(z) g_{\nu}(z, t)$ possède manifestement toutes les propriétés demandées dans le lemme.

C. Q. F. D.

Maintenant nous nous proposons de montrer le théorème 1 .

D'après le lemme 5 , il existe une famille holomorphe $\omega=\left(\omega_{z}\right)$ de différentielles non nulles dans $D(\rho)$. On peut choisir $d(d=2 g+n-1)$ courbes simples fermées régulières $\gamma_{o}^{1}, \ldots, \gamma_{o}^{d}$ dans $D_{o}^{\prime \prime}$, de manière que leurs classes d'homologie forment une base de $\mathrm{H}_{1}\left(D_{o}^{\prime \prime}, \boldsymbol{Z}\right)$, que chacune d'elles coupe une au plus des autres en un seul point et que $K_{o}=\gamma_{o}^{1} \cup \ldots \cup \gamma_{o}^{d}$ ne limite aucun domaine relativement compact dans $D_{o}^{\prime \prime}$. Alors, $K_{o}$ est un ensemble compact tel que $D_{o}$-convexe. D'après le lemme 6 , la variable réelle $t$ étant maintenant un paramètre convenable de l'une de ces courbes, il existe un nombre positif $\rho_{2}<\rho$ et, pour chaque $j=1, \ldots, d$, une fonction $g^{j}(z, p)$ dans $\overline{B\left(\rho_{2}\right)} \times K_{o}$ satisfaisant aux conditions suivantes :

(i) $g^{j}(z, p)$ est continue dans $\overline{B\left(\rho_{2}\right)} \times K_{o}$ et, si l'on fixe $p$, holomorphe par rapport à $z$ sur $\overline{B\left(\rho_{2}\right)}$; 
(ii) $g^{j}(z, p)=0$ quand $p \in \gamma_{o}^{i}(i \neq j)$;

(iii) Pour tout $z \in \overline{B\left(\rho_{2}\right)}$, on a $\int_{T_{z}^{j}} \omega_{z} e^{g_{z}^{j}}=0$ et $\int_{T_{z}^{j}} g_{z}^{j} \omega_{z} e^{g_{z}^{j}} \neq 0$, où $\gamma_{z}^{j}=$ $\left(v^{-1}\left(\gamma_{o}^{j}\right)\right) \cap D_{z}$ et $g_{z}^{j}=\left.g^{j} \circ(\pi \times v)\right|_{r_{z}^{j}}$.

Nous rappelons le nombre $\rho_{1}$ donné dans la condition $5^{\circ}$. D'après le théorème 2, il existe, pour un nombre positif $r<\operatorname{Min}\left(\rho_{1}, \rho_{2}\right)$ et pour chaque $j=1, \ldots, d$, une suite de fonctions holomorphes $\left\{h_{n}^{j}\right\}_{n=1}^{\infty}$ dans $D(\rho)$, qui converge vers $g^{j} \circ(\pi \times v)$ uniformément sur le compact $\left.(\pi \times v)^{-1} \overline{(B(r)} \times K_{o}\right)$. Posons

$$
\phi^{j}(s, z)=\int_{r_{z}^{j}} \omega_{z} \exp \left(s_{1} g_{z}^{1}+\ldots+s_{j} g_{d}^{z}\right),
$$

où $s=\left(s_{1}, \ldots, s_{d}\right)$ est système de $d$ variables complexes. $\phi^{j}(s, z)$ est alors une fonction holomorphe dans $C^{d} \times B\left(\rho_{2}\right)$. Au point $a=(1, \ldots, 1)$ $\in C^{d}$, on a $\phi^{j}(a, z)=0, \frac{\partial \phi^{j}}{\partial s_{i}}(a, z)=0$ pour $(i \neq j)$ et

$$
\frac{\partial \phi^{j}}{\partial s_{j}}(a, z) \neq 0
$$

quel que soit $z \in B\left(\rho_{2}\right)$. Pour tout $z$ fixé dans $B\left(\rho_{2}\right), \Phi_{z}=\phi^{1} \times \ldots \times \phi^{d}$ est alors une application holomorphe $\Phi_{z}: \boldsymbol{C}^{d} \rightarrow \boldsymbol{C}^{d}$ telle qu'on ait $\Phi_{z}(a)=(0, \ldots, 0)$ et que le determinant fonctionnel de $\Phi_{z}$ ne soit pas nul en $a$. D'autre part, $\Phi_{n}^{j}(s, z)=\int_{r_{z}^{j}} \omega_{z} \exp \left(s_{1} h_{n}^{1}+\ldots+s_{d} h_{n}^{d}\right)$ est une fonction holomorphe dans $C^{d} \times B\left(\rho_{2}\right)$. Pour tout compact $L$ dans $C^{d}$, la suite $\left\{\Phi_{n}^{j}\right\}_{n=1}^{\infty}$ converge vers $\phi_{j}(s, z)$ uniformément dans $L \times \overline{B(r)}$. D'où, il existe un entier $n$ suffisamment grand et $d$ fonctions $s(z)=\left(s_{1}(z), \ldots, s_{d}(z)\right)$ holomorphes dans $B(r)$, ayant des valeurs suffisamment voisines de 1 , telles que l'on ait $\phi_{n}^{\jmath}(s(z), z)=0$ dans $B(r)$ pour $j=1, \ldots, d$. Posons, pour $n$ ainsi pris, $f=\left(s_{1} \circ \pi\right) h_{n}^{1}+\ldots+$ $\left(s_{d} \circ \pi\right) h_{n^{d}}$. En vertu de la dernière propriété, pour tout $p \in D_{z}$, l'intégrale $\int_{O_{z}}^{p} \omega_{z} e^{f}$ le long d'un chemin dans $D_{z}$ de $O_{z}$ à $p$ ne dépend pas $\mathrm{du}$ choix du chemin et par suite elle représente une fonction uniforme et holomorphe $h_{z}$ dans $D_{z}$. En faisant varier $z$ dans $B(r)$, on obtient une fonction uniforme $h$ dans $D(r):\left.h\right|_{D_{s}}=h_{z}$. Manifestement, $h$ est holomorphe dans $U(r)=v^{-1}\left(U_{o}\right) \cap D(r)$. D'après le théorème de Hartogs, $h$ est holomorphe dans $D(r)$. Pour toute coordonnée locale 
$u_{z}$ sur $D_{z}$, on a $\frac{\partial h_{z}}{\partial u_{z}} \neq 0$, ce qui montre que l'application $\pi \times h: D(r)$ $\rightarrow B(r) \times C^{1}$ est une immersion voulue. $\quad$ C. Q. F. D.

\section{Références}

[ 1] Gunning, R., and Narasimhan, R., Immersion of open Riemann surfaces, Math. Ann., 174 (1967), 103-108.

[2] Hörmander, L., An Introduction to Complexe Analysis in Several Variables, D. van Nostrand Co. Inc., Princeton, N. J., 1966.

[3] Nishino, T., Nouvelles recherches sur les fonctions entières de plusieurs variables complexes (I), J. Math. Kyoto Univ., 8(1968), 49-100.

[4] ; (II) Fonctions entières qui se réduisent à celle d'une variable, J. Math. Kyoto Univ., 9 (1969), 221-274.

[5] ; (III) Sur quelques propriétés topologiques des surfaces premières, J. Math. Kyoto Univ., 10(1970), 245-271.

[6] ; (IV) Type de surfaces premières, J. Math. Kyoto Univ., 13(1973), 217-272.

[7] Yamaguchi, H., Famille holomorphe de surfaces de Riemann ouvertes, qui est une variété de Stein. à paraître. 